\author{
Bowei Li ${ }^{1}$ \\ Wei Zhang ${ }^{1,2}$ \\ Lingxin Chen ${ }^{1}$ \\ Bingcheng Lin ${ }^{3}$ \\ ${ }^{1}$ Key Laboratory of Coastal Zone \\ Environmental Processes and \\ Ecological Remediation Yantai \\ Institute of Coastal Zone \\ Research, Chinese Academy of \\ Sciences, Yantai, P. R. China \\ ${ }^{2}$ University of the Chinese \\ Academy of Sciences, Beijing, \\ P. R. China \\ ${ }^{3}$ Dalian Institute of Chemical \\ Physics, Chinese Academy of \\ Sciences, Dalian, P. R. China
}

Received March 15, 2013

Revised April 15, 2013

Accepted April 22, 2013
Research Article

\section{A fast and low-cost spray method for prototyping and depositing surface- enhanced Raman scattering arrays on microfluidic paper based device}

In this study, a fast, low-cost, and facile spray method was proposed. This method deposits highly sensitive surface-enhanced Raman scattering (SERS) silver nanoparticles (AgNPs) on the paper-microfluidic scheme. The procedures for substrate preparation were studied including different strategies to synthesize AgNPs and the optimization of spray cycles. In addition, the morphologies of the different kinds of paper substrates were characterized by SEM and investigated by their SERS signals. The established method was found to be favorable for obtaining good sensitivity and reproducible results. The RSDs of Raman intensity of randomly analyzing 20 spots on the same paper or different filter papers depositing AgNPs are both below 15\%. The SERS enhancement factor is approximately $2 \times 10^{7}$. The whole fabrication is very rapid, robust, and does not require specific instruments. Furthermore, the total cost for 1000 pieces of chip is less than $\$ 20$. These advantages demonstrated the potential for growing SERS applications in the area of environmental monitoring, food safety, and bioanalysis in the future.

\section{Keywords:}

Paper microfluidics / Silver nanoparticles / Spray / Surface-enhanced Raman scattering DOI 10.1002/elps.201300138

Additional supporting information may be found in the online version of this article at the publisher's web-site

\section{Introduction}

Since the Raman scattering phenomenon was discovered, scientists from different research areas made a large number of in-depth studies of the theory, and many applications of Raman spectroscopy achieved with fruitful results. In the early stages of study of Raman spectroscopy, the poor power and the monochromatic characteristics of the laser light source limited the development of Raman spectroscopy. To date, the surface-enhanced Raman scattering (SERS) overcomes the shortcoming of low sensitivity of conventional Raman spectroscopy, and can provide more structural information adsorbed on or near a metal surface molecules $[1,2]$. SERS has

Correspondence: Professor Lingxin Chen, Key Laboratory of Coastal Zone Environmental Processes and Ecological Remediation, Yantai Institute of Coastal Zone Research, Chinese Academy of Sciences, Yantai 264003, P. R. China E-mail: Ixchen@yic.ac.cn

Fax: $+86-535-2109130$

Abbreviations: AgNPs, silver nanoparticles; HH, hydroxylamine hydrochloride; PVD, physical vapor deposition; R6G, rhodamine-6G; SERS, surface-enhanced Raman scattering been widely applied to the surface of the study, the interface surface state of the adsorption, biological macromolecules interface [3], and the configuration conformation, etc. [4-7].

The microfluidic technique provides a highly defined space that can address the behavior, control, and manipulation of fluids at volumes on the order of microliters and handle a variety of different reactions easily and accurately $[8,9]$. In addition, other advantages are obvious such as the low sample consumption [1,10,11], faster analysis and response [12,13], massive parallelization and integration $[8,14-16]$. Combining these attractive lab-on-a-chip features with the Raman spectroscopy provides a promising platform for synergistically integrating the variety of implemented functions with a flexible sample control. The sensitive, rapid, and noninvasively detecting results have attracted much attention in recent years $[3,17]$. Many SERS applications on microfluidic platform have been developed [1, 18-21]. Recently developed paper-based microfluidics technology represented an economical and effective way to perform simple operation, easy to transport use. This technology was quickly applied to a series of different areas of research, such as point of care [22-25], SERS

Colour Online: See the article online to view Figs. 1, 2, 4, and 5 in colour. 
[26-28], electrochemical sensor [29-31], and cell culture, etc. [32].

Increasing efforts have been invested into the SERS investigation on the paper's format. Especially, it is critical to prototype nanoparticles on cellulose substrate in a controllable and easy way. Some methods have been proposed for this based on different mechanisms. For instance, White et al. first used an ink printer to realize SERS detection by patterning nanoparticles on the paper substrate with satisfied results [26]. Sun et al. achieved an efficient SERS test strips by simple physical vapor deposition (PVD) coating [33]. Lee and Ngo developed the method by soaking paper into the nanoparticle solutions $[27,34]$. These approaches offer some useful explorations to prototype SERS paper strip.

However, these methods have few limitations. For example, in the printing method the silver nanoparticles (AgNPs) could not be maintained for a long time because these particles are easily oxidized by air. Hence the frequent replacement of nanoparticle solution in the ink cartridge is needed. However, the ink cartridge is usually placed inside the machine that makes the process of replacement very cumbersome. In addition, the aggregation of the AgNPs occurs easily but is hard to observe because of the placement of ink cartridge inside the machine, which leads to the clogging of the printer's nozzles and causes damage to the machine. In the PVD method, the AgNPs can be uniformly distributed on the paper with very stable signal, but the method needs to be equipped with very expensive instrument and sputtering bombardment of a silver or gold target is also very costly. In the dipping method [34], it generally takes a long time (1 or 2 days) to prepare the paper strip by immersing it into the $\mathrm{Ag}$ or $\mathrm{Au}$ nanoparticle solution.

In order to address these issues, to our best knowledge, this work represents the first approach of using spray deposition method to fabricate SERS substrate on the papermicrofluidic device. Compared with the previous methods $[26,33]$, the scheme provides a simple, rapid, and low-cost solution to realize the nanoparticles' deposition on the paper with desired results. The rhodamine-6G (R6G) was used as a model to show this concept. This method does not require a PVD equipment or a professional printer, and the nanoparticle's deposition can be rapidly realize with the help of an inexpensive spray bottle that is very convenient.

\section{Materials and methods}

\subsection{Chemicals and materials}

All chemicals used were of analytical grade. Silver nitrate $\left(\mathrm{AgNO}_{3}, 99.8 \%\right)$, sodium borohydride $\left(\mathrm{NaBH}_{4}, 96 \%\right)$, and sodium hydroxide $(\mathrm{NaOH}, 96 \%)$ were obtained from Sinopharm Chemical Reagent Co. (Shanghai, China). Hydroxylamine hydrochloride $\left(\mathrm{NH}_{2} \mathrm{OH} \cdot \mathrm{HCl}, 98.5 \%\right)$ and trisodium citrate dehydrate $\left(\mathrm{C}_{6} \mathrm{H}_{5} \mathrm{Na}_{3} \mathrm{O}_{7} \cdot 2 \mathrm{H}_{2} \mathrm{O}, 99.0 \%\right)$ were purchased from Guangcheng Chemical Reagent Co. (Tianjin, China). R6G was obtained from Aladdin. Ultrapure water purified with a Milli-Q system ( $18 \Omega$ /'cm resistance) was used in all aqueous solutions and rinsing procedures.

\subsection{Preparation of AgNPs}

The silver colloids used for SERS were similar to that used in the previous reports. Briefly, AgNPs were prepared by two different methods. In one method, silver nitrate was reduced by using hydroxylamine hydrochloride $(\mathrm{HH})$ [35] and in the other method sodium borohydride was used to produce AgNPs, where the citrate was employed as a stabilizer [36, 37]. In the $\mathrm{HH}$ reduction, $90 \mathrm{~mL}$ of $\mathrm{HH}$ solution $\left(1.67 \times 10^{-3} \mathrm{M}\right)$ containing $3.33 \times 10^{-3} \mathrm{M}$ sodium hydroxide was prepared. Then $10 \mathrm{~mL}$ of silver nitrate solution $\left(10^{-2} \mathrm{M}\right)$ was added to this solution and continuously stirred for $1 \mathrm{~h}$. In the preparation of citrate-stabilized AgNPs, $250 \mu \mathrm{L}$ of $100 \mathrm{mM} \mathrm{AgNO}_{3}$ and $250 \mu \mathrm{L}$ of $100 \mathrm{mM}$ trisodium citrate were added to $100 \mathrm{~mL}$ of water while stirring. Then $6 \mathrm{~mL}$ of $5 \mathrm{mM} \mathrm{NaBH}_{4}$ was added to above aqueous solution while stirring. The colloidal silver solution was stirred for another $30 \mathrm{~min}$ and then stored overnight.

Prior to use, the two kinds of colloidal silver solutions were centrifuged at $7000 \mathrm{rpm}$ for $10 \mathrm{~min}$ to concentrate nanoparticles. Ninety-five percent of the upper supernatant was discarded after centrifugation, and glycerol was added to the remaining $5 \mathrm{~mL}$ AgNPs solution to adjust the viscosity and surface tension of colloid on the paper in the volume ratio of 2:5 (glycerol/colloid solution). The glycerol has not had obvious Raman signals after our first test. After the solution was well mixed, the AgNP solution was deposited onto the paper through a spray bottle and air-dried at room temperature.

\subsection{Instruments}

The chip was designed with drawing software (Adobe Illustrator) and then directly printed onto the filter paper (Whatman chromatography No.1 paper, GE) by a wax printer (XEROX Phaser 8560DN) with highest resolution of $2400 \mathrm{dpi}$. After printing, the paper strip was kept in an oven at $150^{\circ} \mathrm{C}$ for $20 \mathrm{~s}$ in order to let the wax penetrate through the paper completely, forming a hydrophobic barrier.

The morphology of the AgNPs on the paper chip was characterized by SEM using a Hitachi-4800 scanning electron microscope operated at an accelerating voltage of $5 \mathrm{kV}$. Hydraulic radius determination was performed by the Zetasizer $3000 \mathrm{HS}$ particle size analyzer instrument (Malvern, UK). SERS spectra were acquired from confocal DXR Raman spectrometer (Thermo Fisher, USA). A $632.8 \mathrm{~nm}$ diode pumped He:Ne laser was equipped with a power of $3.8 \mathrm{~mW}$. An objective lens was used to focus the excitation light onto the cellulose substrate and all spectra were collected with an $8 \mathrm{~s}$ integration time. Using a micropipette, $10 \mu \mathrm{L}$ R6G was placed onto the chip. SERS measurements were acquired after paper was dried and exposed to CCD for $2 \mathrm{~s}$. 

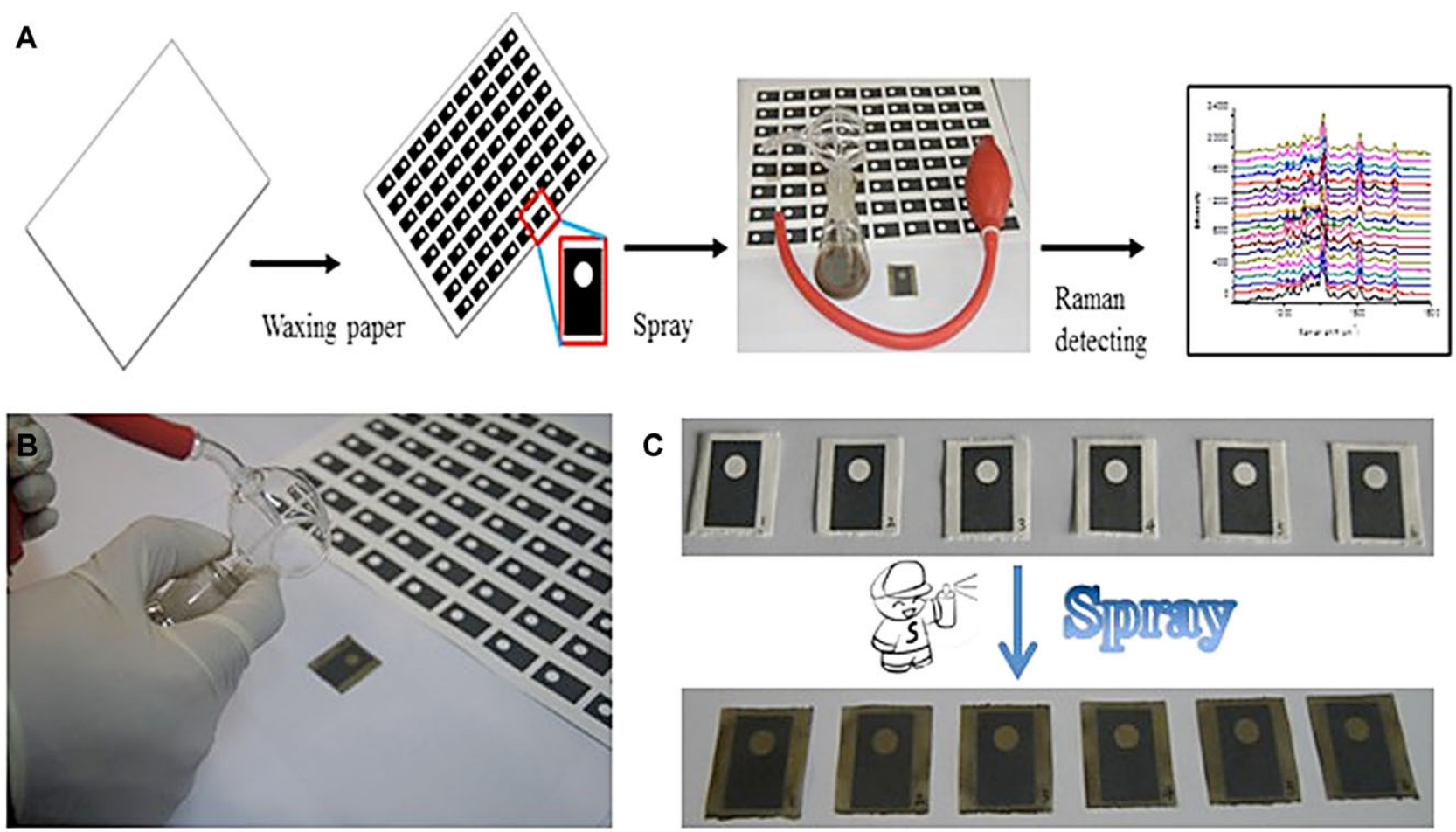

Figure 1. (A) Schematic diagram of the spraying method for fabricating paper-based microfluidic devices in order to use for SERS analysis. (B) Spray AgNPs onto the paper. (C) The same papers before and after spraying AgNPs on its surface.

\section{Results and discussion}

\subsection{Characterization of device and methodology}

The schematic diagram of paper fabrication progress using spray technology is shown in Fig. 1A and B. First, we use printer (XEROX Phaser 8560DN) for designing a structure that contains hydrophobic and hydrophilic areas on the paper chip. The chip's dimension was $35 \mathrm{~mm} \times 15 \mathrm{~mm}$ with a square shape. The circular portion of the intermediate hydrophilic region had a diameter of $6 \mathrm{~mm}$ and the remaining surface was covered by wax. After $20 \mathrm{~s}$ of wax melting, the wax penetrated the paper creating a barrier that blocks the flow of water. The prepared $14 \mathrm{~mL}$ AgNPs solution was contained in the spray bottle that was used to spray the AgNPs onto the cellulose paper kept at a vertical distance of $10 \mathrm{~cm}$ and the air-dried at room temperature (Fig. 1C). We noticed that even if chips' surface was coated with AgNPs, water can be very easily transported in the hydrophilic region, thus maintaining the surface hydrophilic effect. Therefore, when the analyzed sample was added by micropipette, it distributed well in the whole detection area, which guarantees a favorable condition for the following SERS experiments.

Two classic methods using different procedures to employ the synthesis of AgNPs were reported. In one method silver nitrate is reduced by $\mathrm{HH}$ and other method uses sodium borohydride, which is stabilized by citrate (SC). To understand the Raman contributions of these two methods, we made preliminary assessments for their AgNPs, respectively.
The particle size was measured by Malvern particle size analyzer, and the results are shown in Supporting Information Fig. 1. The particle diameter using $\mathrm{HH}$ method, and the sodium borohydride method was $69 \mathrm{~nm}$ and $38 \mathrm{~nm}$, respectively. The preliminary SERS test was performed using R6G in solution (not on paper) and the results are shown in Supporting Information Fig. 2. It has been observed that AgNPs synthesized by HH method had a higher signal than synthesized by the other methods. Therefore, the HH method was chosen for the preparation of AgNPs for following experiments.

\subsection{Comparison with direct dropping method}

After the comparison of different nanoparticles synthesis method, we will need a method to uniformly cover the AgNPs on the paper. Although the dipping method has uniform coverage, but the method of production time is too long, usually require one to two days, and a long period of air exposure will affect the SERS activity of nanoparticles (see subsequent discussion). Therefore we proposed a quick and feasible strategy to deposit AgNPs onto the paper.

Actually, when talking about the spray deposition method, a question naturally asked is whether dropping the AgNPs onto the paper directly is much simple. Due to the coffee ring effect, when a drop solution containing nanoparticles was directly added on a filter paper, the nanoparticles are deposited in the edge region at higher concentration than 

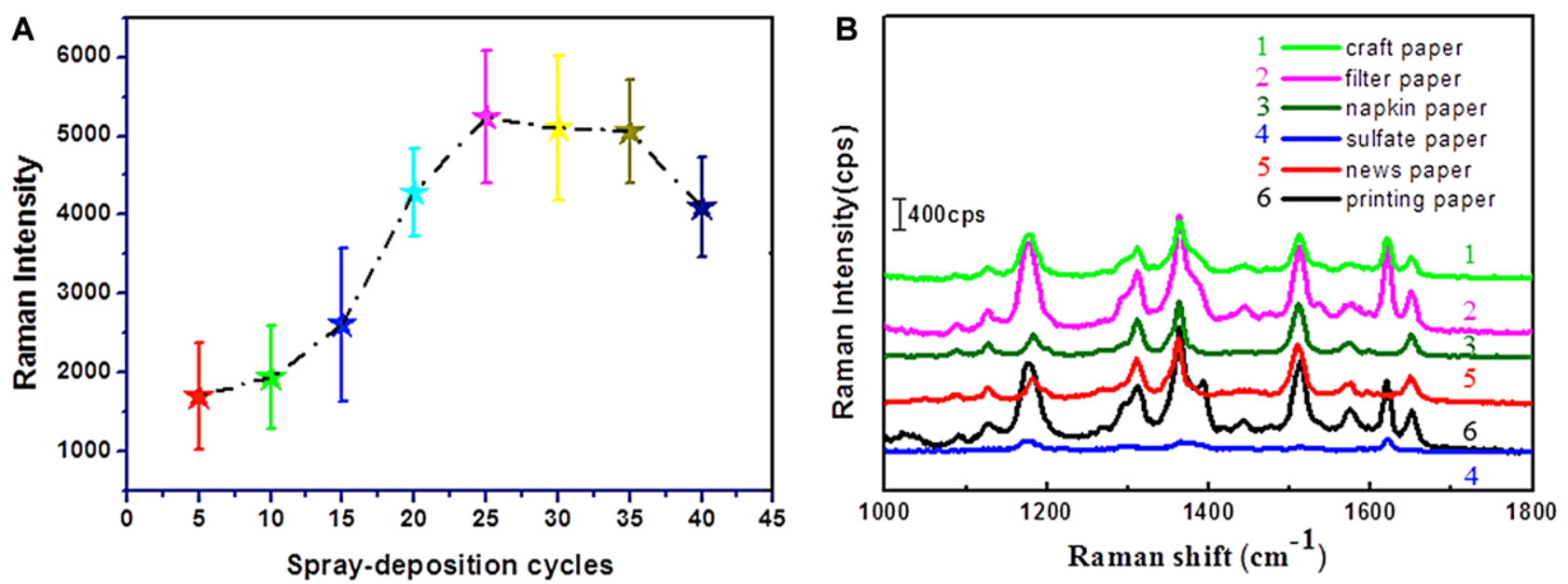

Figure 2. (A) Raman intensity curve for detecting $5 \times 10^{-6} \mathrm{M} \mathrm{R6G}$ by spraying different (from five to 35) cycles of AgNPs on the filter paper. (B) Surface-enhanced Raman scattering spectra obtained from R6G on different cellulose paper. The spray cycles of AgNPs are 25 and the concentration of R6G is $1 \times 10^{-6} \mathrm{M}$.

in the intermediate region [38,39]. While the substrate's or the paper's unevenness also can lead to an irregular flow distribution on the paper, which results in the irregular particle deposition. On the other hand, spray method can produce tiny and well-proportioned shape droplets that could be very effective and convenient to generate uniform distribution on the paper.

To prove this point, we measured two kinds of chips using the direct dropping and spray methods, and randomly selected their 20 different spots, respectively, to measure their Raman signals. The results are displayed in Supporting Information Fig. 3. The RSD for dropping and spray methods are 64.4 and $14.5 \%(n=20)$. As expected for the case of nanoparticle's deposition, the spray method's measurements reveal more reproducible and accurate data. In addition, we also obtained SEM images to illustrate the differences of AgNPs deposition between these two methods. As shown in Supporting Information Fig. 4, it can be easily observed that the depositions are not uniform using dropping method and this result is in agreement with its SERS signals (Supporting Information Fig. 3).

\subsection{Optimization with different spray cycles and on different kinds of paper}

The AgNPs substrate plays a very important role in the SERS signal enhancement. Obviously, the number of AgNPs deposited on scraps of paper is related to the number of spray cycles. In order to make the Raman signal for ideal effect, we investigated about the number of cycles of the spray optimization first. A total of $5 \times 10^{-6} \mathrm{M}$ R6G was used as an example and the laser was excited at $633 \mathrm{~nm}$ wavelength with the power at $3.8 \mathrm{~mW}$. As shown in Fig. 2A, the signal is very tiny at the beginning because the amount of AgNPs deposited onto the paper is relatively small, and it is difficult to form a hot spot effect. With the spray cycles increasing, the signal intensity has an exponential enhancement. It is gener- ally regarded that the high surface density of AgNPs shows more energetic SERS activity. When increased from 25 to 35 cycles, the signal strength reached a plateau and remained saturated. Taking into account that the increase of the spray cycles increases AgNPs, we did not make a further investigation after 35 cycles because it is not cost-effective and was considered wastage of time. The working conditions of spray cycles were chosen as 25 cycles for the following experiments unless otherwise noted.

Recently, paper as a low-cost, easy to transport, and ideal material has attracted much attention. In order to establish the universality of the method, we conducted a test using a variety of different papers and the spray cycles were kept at 25. The commonly used papers include sulfate paper, napkin paper, printing paper, kraft paper, newspaper, and filter paper. Figure 3 provides a photo of AgNPs deposited onto different papers taken under the SEM with the 20 and $2 \mu \mathrm{m}$ scale bars depicting the original SEM images and its enlarged insets, respectively. Although the mechanism for the SERS enhancement is still not completely understood, it is generally regarded that the formation of SERS hot spot plays an important role. Figure 3C, E, and F shows the AgNPs aggregation phenomenon on the printing paper, newspaper, and filter paper, respectively. The formation of silver clusters generates a hot spot effect, which promotes enhanced Raman scattering effect and increases the sensitivity of detection $[2,40]$.

In order to further prove that different papers producing the AgNPs clusters may affect the hot spot effect, a concentration of $1 \times 10^{-6} \mathrm{M}$ R6G's Raman scattering signal was detected on different materials of the paper as shown in Fig. 2B. It can be observed that filter paper and printing paper have strong Raman response. The newspaper, napkin paper, and kraft paper's signal appear weaker than the printing paper and filter paper. The sulfate paper showed smallest Raman intensity. Several successful attempts to perform this process were carried out, which indicates the spray technique could be a versatile nanoparticle deposition method used on 

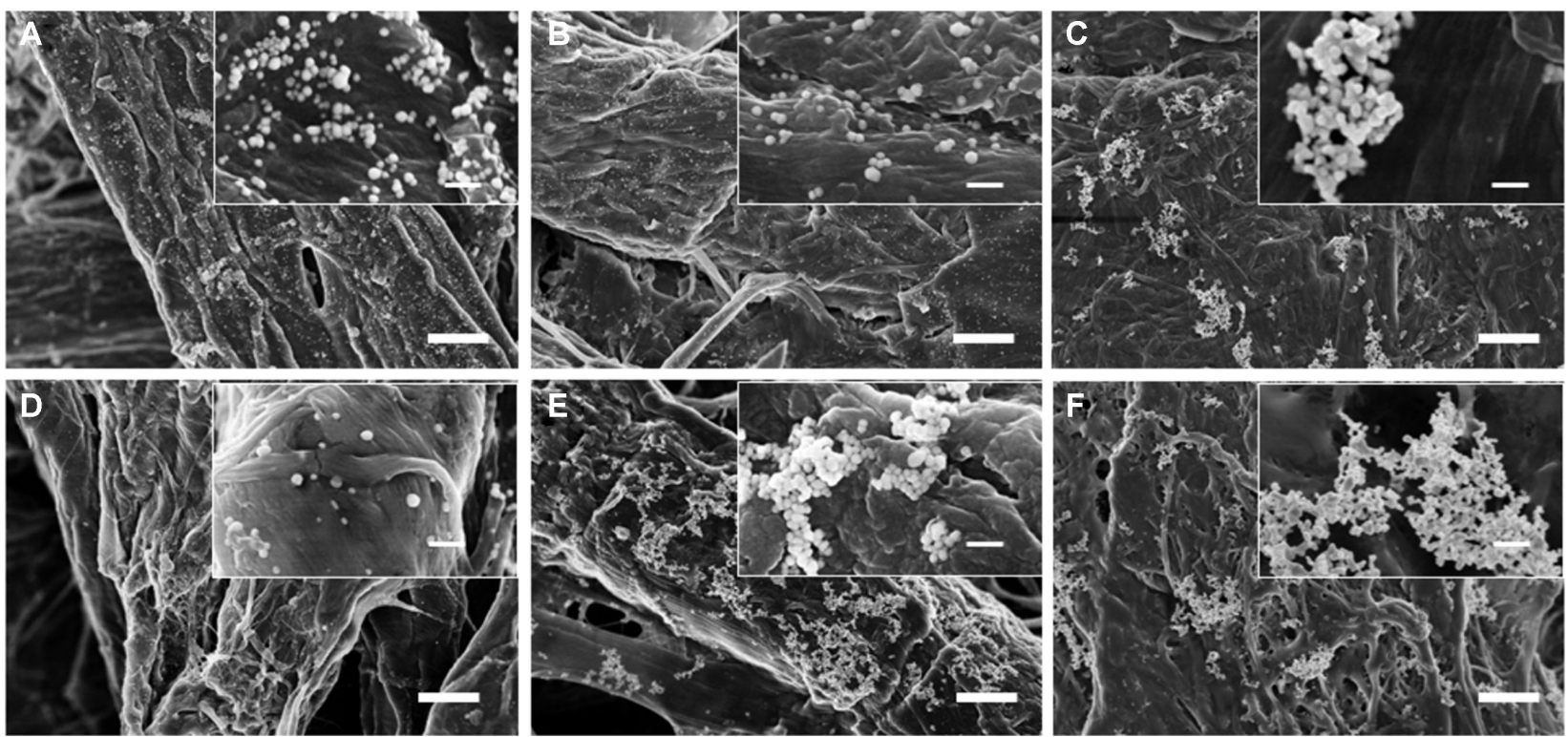

Figure 3. SEM images of different kinds of papers: (A) sulfate paper; (B) kraft paper; (C) printing paper; (D) napkin paper; (E) newspaper; and (F) filter paper. The insets are the enlarged fiber structures of its corresponding papers. The scale bars of the original SEM images and its enlarged insets are 20 and $2 \mu \mathrm{m}$, respectively.

Table 1. The cost of fabricating 1000 pieces of SERS chip $(35 \mathrm{~mm}$ $\times 15 \mathrm{~mm}$

\begin{tabular}{|c|c|c|c|}
\hline $\begin{array}{l}\mathrm{AgNO}_{3} \\
\text { solution }\end{array}$ & $\begin{array}{l}\mathrm{NaOH} \text { and } \\
\mathrm{NH}_{2} \mathrm{OH} \cdot \mathrm{HCl}\end{array}$ & $\begin{array}{l}\text { The filter papers } \\
\left(\text { Whatman }^{\circledR}\right)\end{array}$ & $\begin{array}{l}\text { Spray } \\
\text { bottle }\end{array}$ \\
\hline$\$ 4$ & $\$ 1$ & $\$ 5$ & $\$ 9$ \\
\hline
\end{tabular}

different papers. The cost of reagent to fabricate 1000 chips (not included the expense of spray bottle) is only $\$ 10$ that makes it very cost-effective (shown in Table 1 ). In this approach, the filter paper's attractive characteristics of fluid's easy transportation and water phase emulsion uniformity of diffusion and reaction make filter paper the best choice than others.

\subsection{The method's sensitivity and reproducibility}

For obtaining the good sensitivity and reproducibility, some optimization has been implemented as mentioned above, including the different nanoparticles fabrication routine, the spray cycles, and the various materials of the papers. After completing them, the sensitivity and reproducibility of this method were evaluated by using R6G as a model molecular to explore this strategy. Figure 4A shows the representative Raman spectra of R6G at various concentrations with the paper sprayed by AgNPs for 25 cycles. There are many spectra features that are characteristic of R6G, which can be used for direct quantitative determination, such as those at 1177 , $1300,1360,1580 \mathrm{~cm}^{-1}$. According to the spectra shown, the peak around $1360 \mathrm{~cm}^{-1}$ was the most representative one, and its intensity was very sensitive. Therefore, it was chosen as an ideal peak for the quantitative analysis of R6G. A series of $10 \mu \mathrm{L}$ concentrations of $\mathrm{R} 6 \mathrm{G}$ ranging from $10^{-9}$ to $10^{-5} \mathrm{M}$ were introduced to the hydrophilic region and the results are illustrated in Fig. 4B. Data represent the average of measurements from four different detection spots. The values showed a good quantitative agreement with the results determined by dipping or printing method reported in the literature.

In the present study, the analytical SERS enhancement factor was calculated using the following formula:

$\mathrm{EF}=\left(I_{\mathrm{SERS}} / I_{\mathrm{S}}\right) \times\left(C_{\mathrm{S}} / C_{\mathrm{SERS}}\right)$,

where $\boldsymbol{C}_{\mathrm{S}}$ is the concentration of the analyte solution that produces a spontaneous Raman signal, and $\boldsymbol{C}_{\mathrm{SERS}}$ is the concentration of analyte analyzed on an SERS substrate. The $\boldsymbol{I}_{\mathbf{S}}$ and $\boldsymbol{I}_{\text {SERS }}$ represent their Raman signals under the above experimental conditions, respectively. For a $10 \mathrm{mM}$ R6G without SERS amplification effect, the acquired SERS signal ( $n=4$ ) versus $1360 \mathrm{~cm}^{-1}$ was approximately equal to the signal obtained for $5 \times 10^{-10} \mathrm{M}$ R6G on the paper deposited with AgNPs. This indicated that the enhancement factor was $2 \times 10^{7}$.

As the paper substrate had more random irregular structure than glass or other substrate, to keep consistency in measured signal intensity will be an important issue for SERS analysis. Therefore, the reproducibility of Raman performance was evaluated at different locations and in different papers. In Supporting Information Fig. 3, 20 randomly deposited SERS active spots on the same paper were investigated and the RSD result was fine (14.5\%). Supporting Information Fig. 5 showed its corresponding SERS spectra respectively. Meanwhile, we also recorded SERS signals by analyzing $5 \times 10^{-6} \mathrm{M}$ R6G on the 20 different filter papers deposited with AgNPs. As illustrated in Fig. 5A, there were no 
A

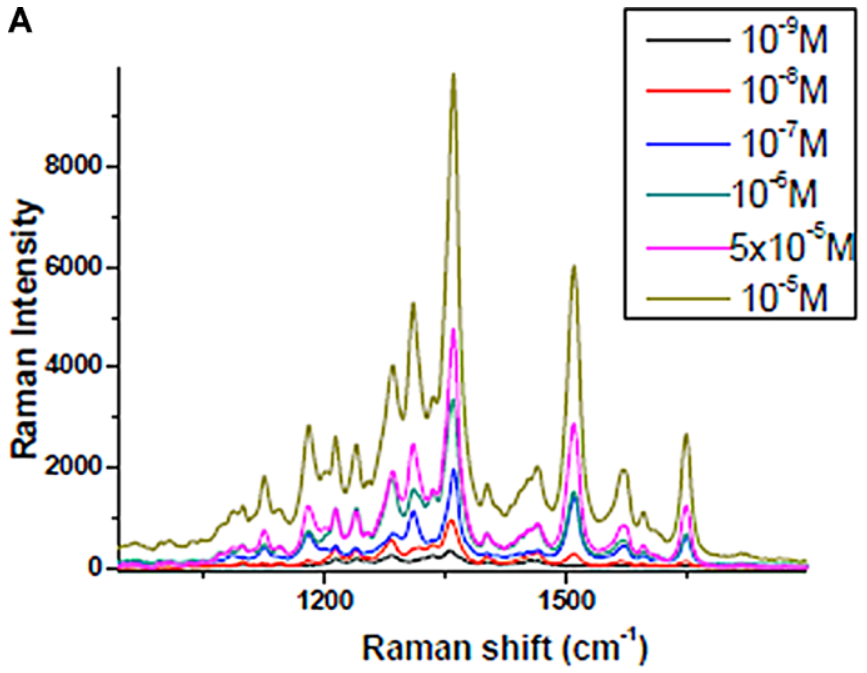

B

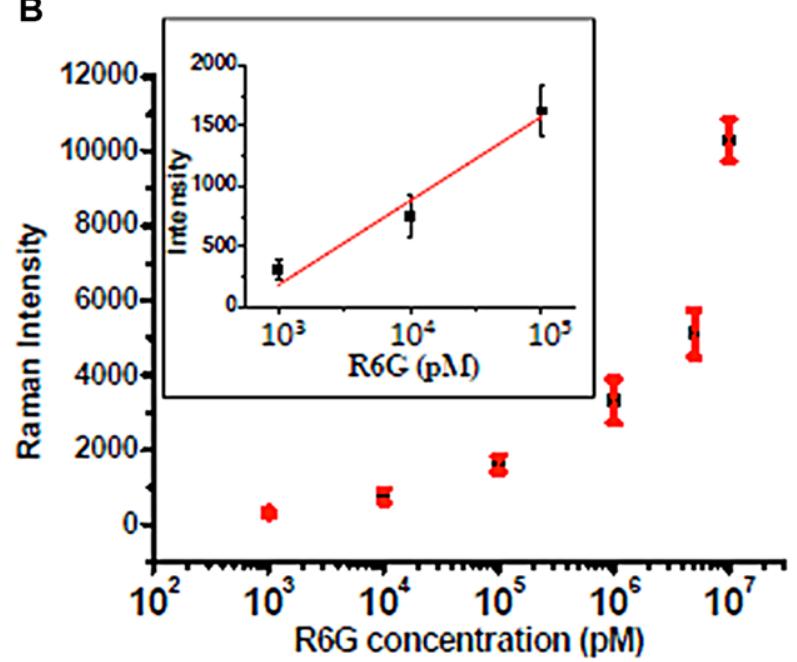

Figure 4. (A) A set of representative SERS spectra with different R6G concentrations on the silver nanostructured paper. The R6G concentrations are $10^{-9}, 10^{-8}, 10^{-7}, 10^{-6}, 5 \times 10^{-6}, 10^{-5} \mathrm{M}$. (B) Average SERS signal magnitude and its error bar $(N=4)$ for each R6G concentration. Inset: the enlarged portion of the plot in the R6G concentration range of $10^{3}-10^{5} \mathrm{pM}$. The regression equation is $Y=-698 \times \log _{10}(X)-1915, R^{2}=0.94$.

significant differences observed between 20 curves and the SDs were only $14.2 \%$. The experimental deviation is mainly coming from the irregular paper structure, herein, the RSD (approximately 15\%) is reasonable. These results revealed that the spraying deposition of AgNP is favorable to obtain a good and stable performance.

\subsection{The investigation of SERS activity change with time}

To test the SERS activity of silver nanostructure obtained by spray deposition, we investigated its relationship to the time change by using fresh spray strips without $\mathrm{N}_{2}$ atmosphere protection. It was clearly observed in Fig. 5B that there were no obvious changes for Raman intensity during the first $9 \mathrm{~h}$. When the time extended to nearly $14 \mathrm{~h}$, the Raman signal decreased. But when it was exposed in the air for more than one day, the strip's Raman activity decreased and was hardly used for SERS detection. The results are in agreement with the previous report [33]. This phenomenon indicates that the SERS strip needs to be fresh or well-protected by nitrogen or other inert gas. Though the long exposure time to the air will result in the loss of SERS activity, our spray process provides a facile way to fabricate fresh strip. SERS strips are easily available and overcome the limitation of the oxidation of AgNPs. Herein, this advantage will show more potential to SERS strips research.
A

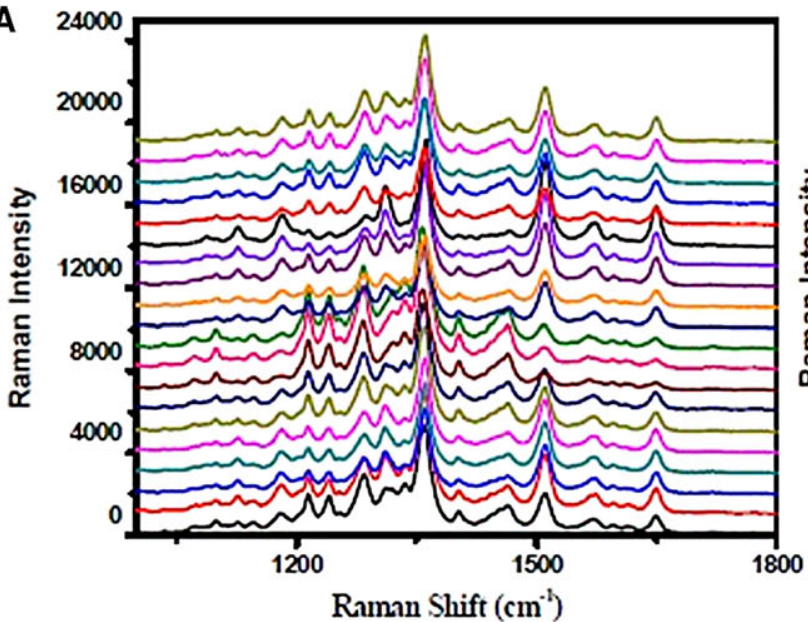

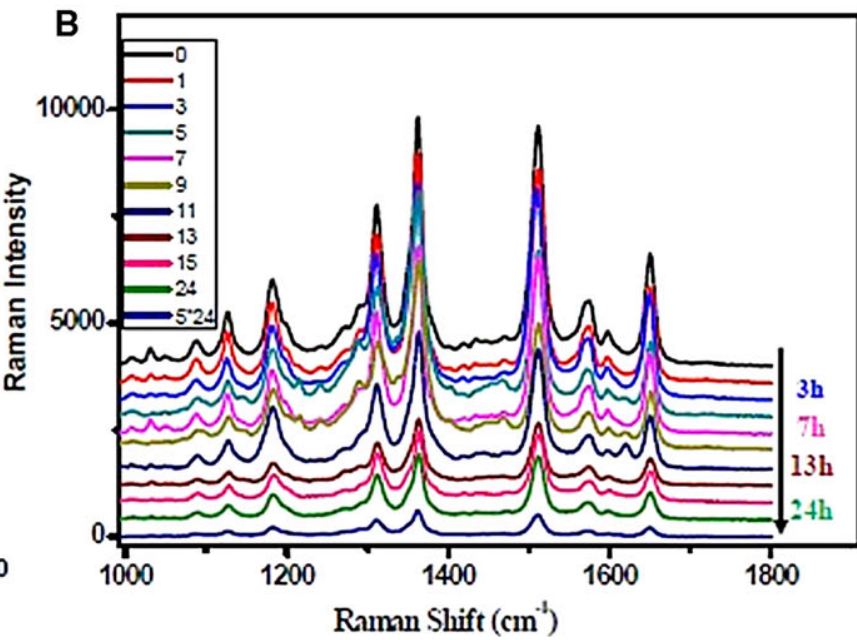

Figure 5. (A) The Raman spectra for evaluating the reproducibility of Raman performance on 20 different papers using $5 \times 10^{-6} \mathrm{M}$ R6G. (B)The SERS intensity change with the time using fresh-made test strips. The time changes were $1,3,5,7,9,11,13,15,24$, and 120 h, respectively. 


\section{Concluding remarks}

In this article, a novel low-cost, rapid, and feasible fabrication method of AgNPs assays on the paper-microfluidic format for SERS detection has been proposed. Compared with previous methods, this method does not need specific or expensive equipment and avoid tedious progresses that enable people to fabricate high performance SERS strips quickly and feasibly in any lab. Using this method, we can achieve the high-sensitivity detection of $10^{-9} \mathrm{M} \mathrm{R6G}$ with good reproducibility and its RSDs are below $15 \%$ on different papers or different locations. More important, the total cost of 1000 pieces of paper can be reduced to less than $\$ 20$. Owing to the paper's attractive features such as low-cost, lightweight, and easy-to-perform detection, this simple spray strategy will be a prospective candidate for the SERS applications such as environmental monitoring, food safety, and rapid analysis detection.

This work was supported by the National Science Foundation of China (21205131, 21275158), the National Special Fund for the Development of Major Research Equipment and Instruments (2011YQ03012404), and the Science and Technology Development Plan of Yantai (2011071). The authors thank Dr. Yunqing Wang for useful discussions.

The authors have declared no conflict of interest.

\section{References}

[1] Wang, G., Lim, C., Chen, L., Chon, H., Choo, J., Hong, J., deMello, A. J., Anal. Bioanal. Chem. 2009, 394, 1827-1832.

[2] Cialla, D., Maerz, A., Boehme, R., Theil, F., Weber, K., Schmitt, M., Popp, J., Anal. Bioanal. Chem. 2012, 403, 27-54.

[3] Wang, Y., Yan, B., Chen, L., Chem. Rev. 2013, 113, 1391-1428.

[4] Nie, S. M., Emery, S. R., Science 1997, 275, 1102-1106.

[5] Kneipp, J., Kneipp, H., Kneipp, K., Proc. Natl. Acad. Sci. USA 2006, 103, 17149-17153.

[6] Kneipp, K., Wang, Y., Kneipp, H., Perelman, L. T., Itzkan, I., Dasari, R., Feld, M. S., Phys. Rev. Lett. 1997, 78, 1667-1670.

[7] Li, J., Chen, L., Lou, T., Wang, Y., ACS Appl. Mater. Interfaces 2011, 3, 3936-3941.

[8] Thorsen, T., Maerkl, S. J., Quake, S. R., Science 2002, 298, 580-584.

[9] Whitesides, G. M., Nature 2006, 442, 368-373.

[10] Teh, S.-Y., Lin, R., Hung, L.-H., Lee, A. P., Lab Chip 2008, $8,198-220$.

[11] Zeng, S., Li, B., Su, X. O., Qin, J., Lin, B., Lab Chip 2009, 9, 1340-1343.

[12] Han, Z., Li, W., Huang, Y., Zheng, B., Anal. Chem. 2009, $81,5840-5845$.

[13] Li, B., Jiang, L., Wang, Q., Oin, J., Lin, B., Electrophoresis 2008, 29, 4906-4913.
[14] Choi, D., Kang, T., Cho, H., Choi, Y., Lee, L. P., Lab Chip 2009, 9, 239-243.

[15] Li, B., Jiang, L., Xie, H., Gao, Y., Qin, J., Lin, B., Electrophoresis 2009, 30, 3053-3057.

[16] Shen, F., Du, W., Davydova, E. K., Karymov, M. A., Pandey, J., Ismagilov, R. F., Anal. Chem. 2010, 82, 4606-4612.

[17] Chen, L., Choo, J., Electrophoresis 2008, 29, 1815-1828.

[18] Choi, N., Lee, K., Lim, D. W., Lee, E. K., Chang, S.-I., Oh, K. W., Choo, J., Lab Chip 2012, 12, 5160-5167.

[19] Lee, M., Lee, K., Kim, K. H., Oh, K. W., Choo, J., Lab Chip 2012, 12, 3720-3727.

[20] Zhou, J., Ren, K., Zhao, Y., Dai, W., Wu, H., Anal. Bioanal. Chem. 2012, 402, 1601-1609.

[21] Chung, E., Gao, R., Ko, J., Choi, N., Lim, D. W., Lee, E. K., Chang, S.-I., Choo, J., Lab Chip 2013, 13, 260-266.

[22] Martinez, A. W., Bioanalysis 2011, 3, 2589-2592.

[23] Lewis, G. G., DiTucci, M. J., Baker, M. S., Phillips, S. T., Lab Chip 2012, 12, 2630-2633.

[24] Lu, Y., Shi, W., Jiang, L., Qin, J., Lin, B., Electrophoresis 2009, 30, 1497-1500.

[25] Lu, Y., Shi, W., Qin, J., Lin, B., Anal. Chem. 2010, 82, 329-335.

[26] Yu, W. W., White, I. M., Anal. Chem. 2010, 82, 9626-9630.

[27] Lee, C. H., Hankus, M. E., Tian, L., Pellegrino, P. M., Singamaneni, S., Anal. Chem. 2011, 83, 8953-8958.

[28] Aldeanueva-Potel, P., Correa-Duarte, M. A., AlvarezPuebla, R. A., Liz-Marzan, L. M., ACS Appl. Mater. Interfaces 2010, 2, 19-22.

[29] Yu, J., Ge, L., Huang, J., Wang, S., Ge, S., Lab Chip 2011, $11,1286-1291$.

[30] Yu, J., Wang, S., Ge, L., Ge, S., Biosens. Bioelectron. 2011, 26, 3284-3289.

[31] Nie, Z., Nijhuis, C. A., Gong, J., Chen, X., Kumachev, A., Martinez, A. W., Narovlyansky, M., Whitesides, G. M., Lab Chip 2010, 10, 477-483.

[32] Derda, R., Tang, S. K. Y., Laromaine, A., Mosadegh, B., Hong, E., Mwangi, M., Mammoto, A., Ingber, D. E., Whitesides, G. M., PLoS One 2011, 6, e18940.

[33] Zhang, R., Xu, B.-B., Liu, X.-Q., Zhang, Y.-L., Xu, Y., Chen, Q.-D., Sun, H.-B., Chem. Commun. 2012, 48, 5913-5915.

[34] Ngo, Y. H., Li, D., Simon, G. P., Garnier, G., Langmuir 2012, 28, 8782-8790.

[35] Leopold, N., Lendl, B., J. Phys. Chem. B 2003, 107, 5723-5727.

[36] Doty, R. C., Tshikhudo, T. R., Brust, M., Fernig, D. G., Chem. Mater. 2005, 17, 4630-4635.

[37] Wei, H., Chen, C., Han, B., Wang, E., Anal. Chem. 2008, $80,7051-7055$

[38] Soltman, D., Subramanian, V., Langmuir 2008, 24, 2224-2231.

[39] Deegan, R. D., Bakajin, O., Dupont, T. F., Huber, G., Nagel, S. R., Witten, T. A., Nature 1997, 389, 827-829.

[40] Lee, S. J., Morrill, A. R., Moskovits, M., J. Am. Chem. Soc. 2006, 128, 2200-2201. 